\title{
Treatment on arrhythmia electric storm in a Jervell and Lange-Nielsen syndrome patient by ablation of the triggering premature ventricular contraction: a case report
}

\author{
Ruiqi Zhang, Chandong Ding, Hui Wang \\ Department of Geriatric Cardiovascular Disease, Second Affiliated Hospital of Anhui Medical University, Hefei, China \\ Correspondence to: Chandong Ding. Department of Geriatric Cardiovascular Disease, Second Affiliated Hospital of Anhui Medical University, No. \\ 678, Furong Road, Hefei, China. Email: chandongding1@sina.com.
}

\begin{abstract}
Jervell and Lange-Nielsen syndrome (JLNS) is a subtype of congenital long QT syndrome (LQTS), which is a potentially life-threatening ion channelopathy characterized by delayed myocardial repolarization that leads to QT prolongation. Since JLNS is a rare disease, there have been few in-depth studies on this disease since yet. Previous studies have found that the pathogenesis of JLNS is related to KCNQ1 or KCNE1 gene mutation. There are a relatively small number of studies reporting the successful ablation treatment on JLNS patient due to the extremely low incidence rate. We report a 51-year-old female patient diagnosed with JLNS through gene detection. The patient has A to G missense mutation in KCNE1 gene, and a subunit with abnormal structure, which is the basis for congenital deafness. The main clinical manifestation of this patient is frequent fatal ventricular arrhythmia triggered by premature ventricular contraction (PVC). Ablation was successfully performed to eliminate the triggering PVC. The patient was not able to have implantable cardioverter defibrillator (ICD) implantation due to economic reasons. During the 12-month follow-up, no syncope or fatal arrhythmia was found in this patient. Our case showed that radiofrequency catheter ablation (RFCA) was an effective way to treat fatal arrhythmia in JLNS patient.
\end{abstract}

Keywords: Jervell and Lange-Nielsen syndrome (JLNS); long QT syndrome; ablation; case report

Submitted Nov 06, 2019. Accepted for publication Sep 02, 2020.

doi: $10.21037 /$ apm-19-460a

View this article at: http://dx.doi.org/10.21037/apm-19-460a

\section{Introduction}

Congenital long QT syndrome is a family hereditary disease, which clinically manifested as abnormally prolonged QT interval, recurrent fatal arrhythmias that lead to syncope and even sudden death, with or without the loss of hearing. LQTS patient without hearing loss is also called Romano-Ward Syndrome (RWS), which is autosomal dominant inherited. Other patients who are autosomal recessive inherited and have hearing loss are considered as JLNS patients, which is much rarer than RWS. The main symptoms of JLNS are syncope and fatal arrhythmia like other LQTS, but it has the unique phenotype of profound bilateral sensorineural hearing loss (1). It has a morbidity of $1-6: 1,000,000$, and a higher possibility of suffering from syncope and sudden death $(2,3)$. Since JLNS is a rare disease, there have been few in-depth studies on this disease since yet. Previous studies have found that the pathogenesis of JLNS is related to KCNQ1 or KCNE1 gene mutation, but fewer studies reported the catheter ablation treatment on JLNS (4-6). The first-line therapy in preventing syncope, cardiac arrest, and sudden death is a betablockade. Propranolol and nadolol have been shown to be superior to metoprolol in preventing cardiac events, but the current consensus is that nadolol as the most preferred (7). In patients with a history of cardiac arrest, an implantable cardioverter-defibrillator (ICD) should be placed. Other indications for ICD placement included patients who are deemed high risk (8). These include a QTc interval greater 


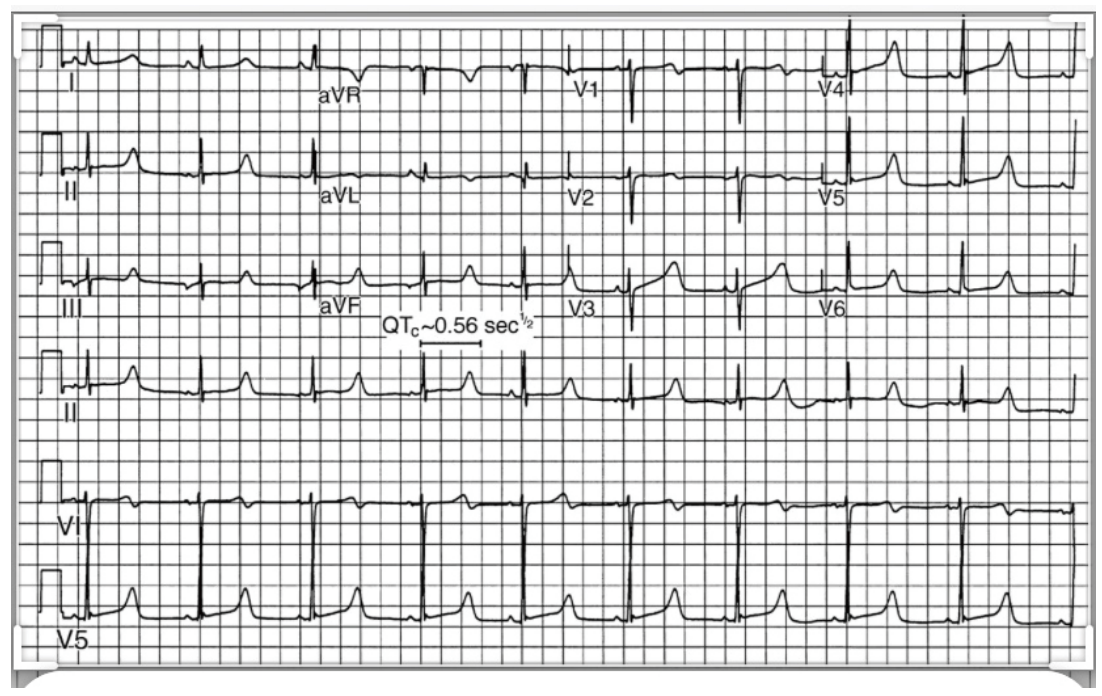

Figure 1 The ECG showed a prolonged QT interval of more than $500 \mathrm{~ms}$.

than $550 \mathrm{~m}$ sec, syncope before age 5 , and a male older than 20 years with the KCNQ1 pathogenic variant (8).

This case in our study showed that the ablation of the triggering premature ventricular contractions (PVCs) could have a positive effect on prevention of fatal arrhythmias in JLNS patients. We present the following case in accordance with the CARE reporting checklist (available at http:// dx.doi.org/10.21037/apm-19-460a).

\section{Case presentation}

A 51-year-old woman was admitted to the Second Affiliated Hospital of Anhui Medical University for repeated syncope 4 years. The patient had been treated with Amiodarone $0.2 \mathrm{~g}$, t.i.d and Valproate $200 \mathrm{mg}$ t.i.d. in Apr 2016 for one month. The patient suffered from palpitation and syncope intermittently, which appeared under fatigue and emotional stimulation. When the syncope occurred, he had limb convulsions, his eyes turned up, his teeth were closed, urine incontinence occurred, and his consciousness was lost. He was conscious after about 10 seconds. After admitted to our department on Aug 31, 2017, myocardial enzymes test like creatine kinase-MB, myoglobin and troponin I were all examined and in normal range. Echocardiogram showed a normal cardiac structure and function. However, the 24-hour ECG monitoring recorded several times of torsades de pointes (Tdp) and even ventricular fibrillation (VF) which was triggered by premature ventricular contraction, and relieved without further medical intervention. Electrocardiogram on Sep 1, 2017 at onset of syncope showed a prolonged QT interval of more than 500 ms (Figure 1). Otorhinolaryngology specialist examination indicated bilateral severe sensorineural deafness. Therefore, the patient was initially diagnosed with JLNS due to the following reasons: (I) fissura auris congenital; (II) QTc >460 ms; (III) the history of syncope/Tdp/ventricular fibrillation. Then the gene sequencing was done on the patient and the patient's family member to confirm the diagnosis of the JLNS from the point view of genetics. The blood of the patient and 9 other family members were tested (Figure 2). After the procedure of acquisition of genome DNA, genomic polymerase chain reaction and DNA sequencing, a missense mutation of A to $G$ was found at position 112 of KCNE1 gene (Figure 3), which led to the consequences that the serine codon (AGT) was replaced by the glycine codon (GGT), and 7 of the 9 family members of the patient all had the same missense mutation of $A$ to $G$ in the same position (Figure 4). Therefore, JLNS was finally diagnosed.

Radiofrequency catheter ablation (RFCA) was considered to be an effective way to stop the fatal arrhythmias, since there was no evidence with high quality to confirm the efficiency of routine drug therapy on JLNS, the continuing Tdp or even VF threatening the life of the patient. It has been reported that RFCA was successful in eliminating the premature ventricular complexes and subsequent polymorphic ventricular tachycardia and prolonged QT (9). The 24 h Holter monitoring before the RFCA 


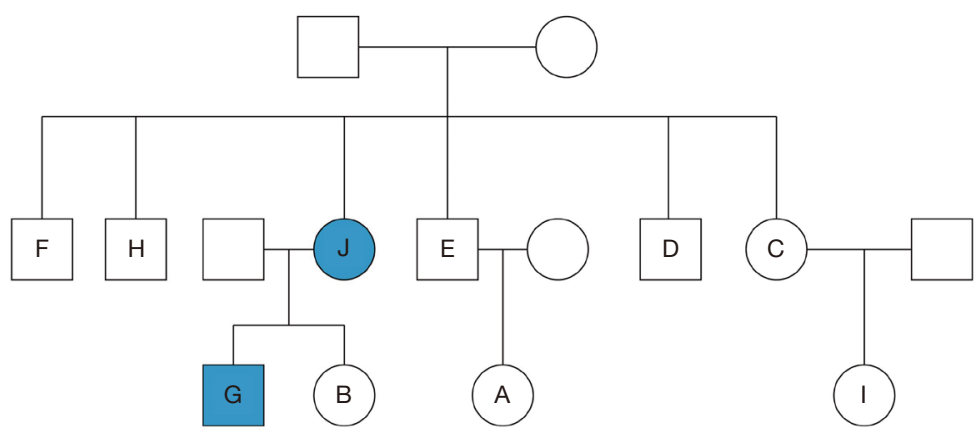

Figure 2 Pedigree of familial diseases. The circle represents female and the square represents male. The letters in the figure are represented by J: the proband; C: the proband's elder sister; G: the proband's son; F: the proband's eldest brother; H: the proband's second brother; E: the proband's third brother; D: the proband's younger brother; B: the proband's daughter; A: the third brother's daughter; I: the elder sister's daughter.

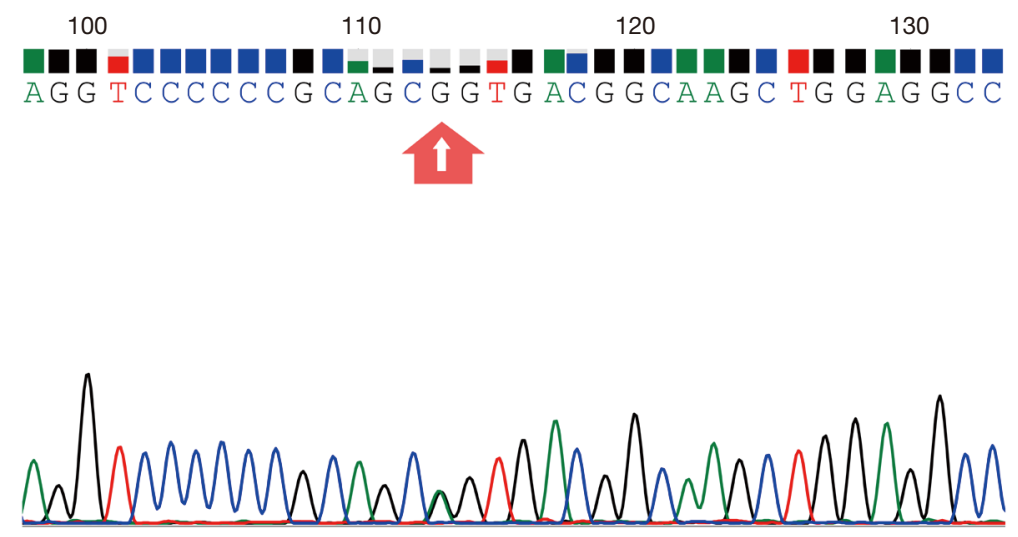

Figure 3 Gene sequencing showed an A to G missense mutation in the patient.

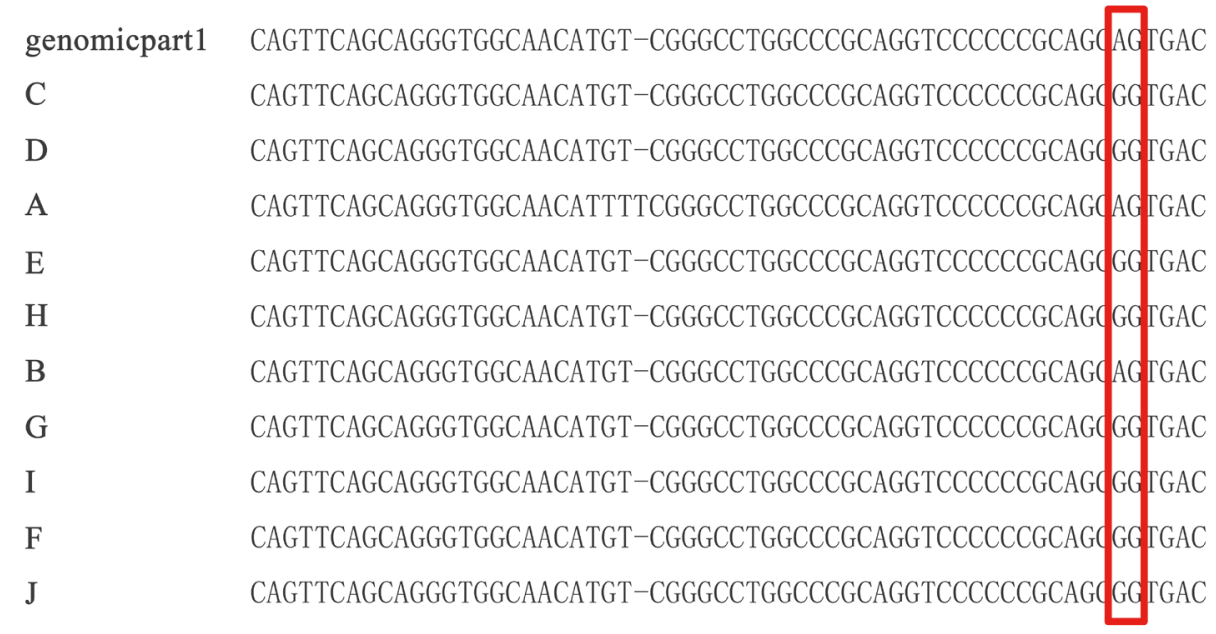

Figure 4 The mutation situation in Exon 112 of the patient and family members ("J" stands for the patient herself and others are other family members). 


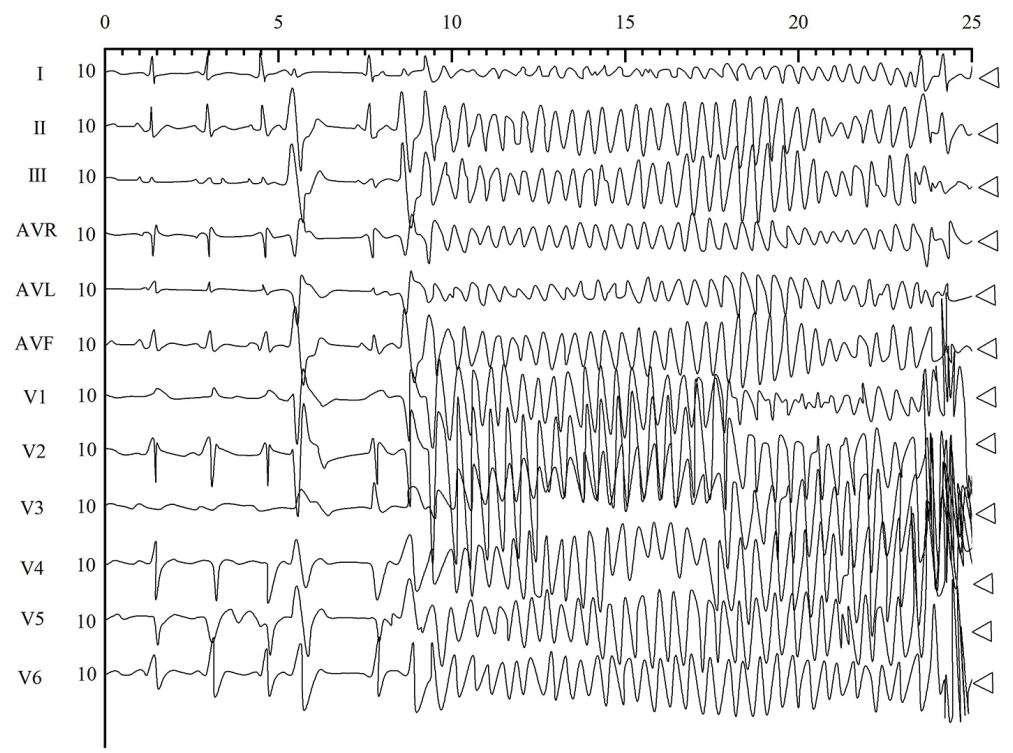

Figure 5 After the use of isoprenaline, ventricular premature beat with the same waveform was recorded.

didn't found many premature ventricular contractions, which makes activation mapping relatively hard and at the first 30 minutes of the operation no ventricular premature beat was observed. RFCA was then performed on Sep 1, 2017. In current case, the isoprenaline was used, then the ventricular premature beat appeared and wide QRS tachycardia was induced (Figure 5). Pacing with ablation catheter at the septum of the right ventricular outflow tract produced QRS morphology perfectly matched with the spontaneous PVCs, then the application was performed at the temperature of $55-60{ }^{\circ} \mathrm{C}$ with a maximum power of 50 watts. No PVCs were observed by ventricular stimuli even with isoproterenol. The patient was not able to have implantable cardioverter defibrillator (ICD) implantation due to economic reasons. The patient was discharged from hospital on September 8, 2017. During the 12-month follow up, no syncope or fatal arrhythmia was observed (Figure 6).

All procedures performed in studies involving human participants were in accordance with the ethical standards of The Second Affiliated Hospital of Anhui Medical University and with the Helsinki Declaration (as revised in 2013). Written informed consent was obtained from the patient for publication of this study and any accompanying images.

\section{Discussion}

KCNE1 gene was firstly reported by Murai in 1989.
The gene located at 21q22.1-22.2, and the length was $40 \mathrm{bp}$ (10). It has three exons, two of them located at 5'UTR. minK protein which is coded by KCNE1 gene is consisted of 129 amino acid residues and include three parts which are extracellular region, transmembrane area and cytoplasm area. KCNE1 gene is expressed in a variety of human tissues and peripheral blood leukocytes. The main working mechanism of minK protein is helping $\alpha$ subunits and $\beta$ subunits to build composite ion gated channel and enhance the stability of composite ion channels through the regulation of $\alpha$-subunit gating. The current dynamic characteristics of KCNE1 in hetero-expression system are similar to those of IKs, the abnormal express of KCNE1 can cause malignant ventricular arrhythmias, recurrent syncope or even sudden death (11-13). The congenital deafness is an important difference in phenotype between JLNS and RWS. The mechanism of the cause of deafness is the formation of abnormal submits in striated cells in the inner ear caused by gene mutation of KCNQ1 or KCNE1 gene, causing secretory disorder of lymphoid system and collapse of cochlear middle ventricle, which leads to deafness (14-16). In our case, the patient has A to G missense mutation in KCNE1 gene, and a subunit with abnormal structure, which is the basis for congenital deafness.

In all, the treatment options for JLNS are minimal. In 2006, a first large study of the JLNS was published, which involved 186 JLNS patients. They found that early therapy with implanted cardioverter/defibrillators must 


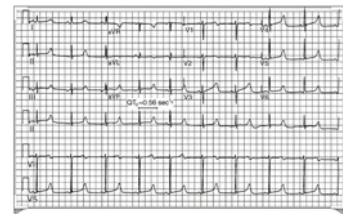

Electrocardiogram at onset of syncope showed a prolonged QT interval of more than $500 \mathrm{~ms}$. Patient was initially diagnosed with JLNS.

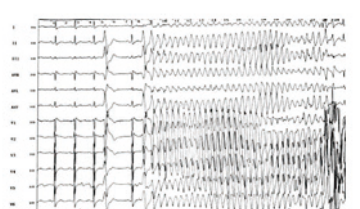

Isoprenaline was used, then the ventricular premature beat appeared and wide QRS tachycardia was induced.

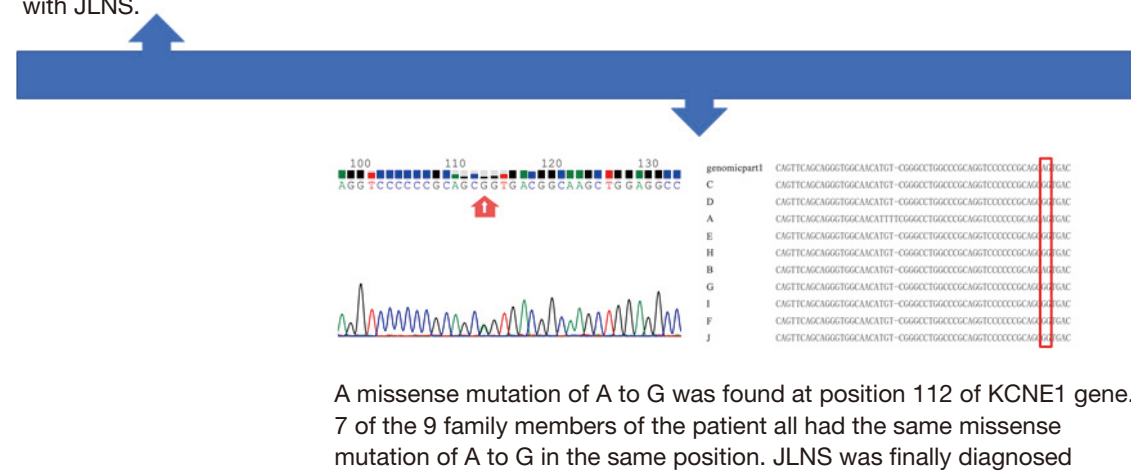

Figure 6 The timeline of the diagnosis and treatment of the patient with JLNS. JLNS, Jervell and Lange-Nielsen syndrome.

be considered and beta-blockers have limited efficacy (8). Another previous study has evaluated the safety and effect of pacing in addition to $\beta$-blocker treatment in children with JLNS. They found that no arrhythmic events occurred in six very young JLNS patients with atrial pacing in combination with increased doses of $\beta$-blockers during the seven years of follow-up, which indicated that this treatment may prevent life-threatening arrhythmias in this high-risk patient group, when left cardiac sympathetic denervation is not available (17).

This is a report on the rare disease. The gene mutation of JLNS was also detected in this study. Moreover, PVCs was successful applicated in treating this kind patient. There were also some limitations in this study. First, this is a case report. The effective of the treatment of PVCs still need to be evaluated in further studies. The other seven family members of the patients were not administrated with systematic examination, though the gene detection was performed.

In conclusion, up till now, there are a relatively small number of studies reporting the successful ablation treatment on JLNS patient due to the extremely low incidence rate. RFCA turns to be an important therapeutic tool for LQTS, especially for JLNS because of the limited effect of drug therapy. Our case showed that RFCA was an effective way to prevent the occurrence of fatal arrhythmia in JLNS patient through the elimination of the triggering PVC.

\section{Acknowledgments}

Funding: None.

\section{Footnote}

Reporting Checklist: The authors have completed the CARE Reporting Checklist. Available at http://dx.doi. org/10.21037/apm-19-460a

Conflicts of Interest: All authors have completed the ICMJE uniform disclosure form (available at http://dx.doi. org/10.21037/apm-19-460a). The authors have no conflicts of interest to declare.

Ethical Statement: The authors are accountable for all aspects of the work in ensuring that questions related to the accuracy or integrity of any part of the work are appropriately investigated and resolved. All procedures performed in studies involving human participants were in accordance with the ethical standards of The Second Affiliated Hospital of Anhui Medical University and with the Helsinki Declaration (as revised in 2013). Written 
informed consent was obtained from the patient for publication of this study and any accompanying images.

Open Access Statement: This is an Open Access article distributed in accordance with the Creative Commons Attribution-NonCommercial-NoDerivs 4.0 International License (CC BY-NC-ND 4.0), which permits the noncommercial replication and distribution of the article with the strict proviso that no changes or edits are made and the original work is properly cited (including links to both the formal publication through the relevant DOI and the license). See: https://creativecommons.org/licenses/by-nc-nd/4.0/.

\section{References}

1. Zhang WJ, Sun Y, Kong WJ. Jervell and Lange-Nielsen syndrome. Lin Chung Er Bi Yan Hou Tou Jing Wai Ke Za Zhi 2019;33:825-9.

2. Schwartz PJ, Crotti L, Insolia R. Long-QT syndrome: from genetics to management. Circ Arrhythm Electrophysiol 2012;5:868-77.

3. Splawski I, Timothy KW, Sharpe LM, et al. $\mathrm{Ca}(\mathrm{V}) 1.2$ calcium channel dysfunction causes a multisystem disorder including arrhythmia and autism. Cell 2004;119:19-31.

4. Schmitt N, Schwarz M, Peretz A, et al. A recessive C-terminal Jervell and Lange-Nielsen mutation of the KCNQ1 channel impairs subunit assembly. Embo J 2000;19:332-40.

5. Splawski I, Shen J, Timothy KW, et al. Genomic structure of three long QT syndrome genes: KVLQT1, HERG, and KCNE1. Genomics 1998;51:86-97.

6. Vyas B, Puri RD, Namboodiri N, et al. KCNQ1 mutations associated with Jervell and Lange-Nielsen syndrome and autosomal recessive Romano-Ward syndrome in Indiaexpanding the spectrum of long QT syndrome type 1 . Am J Med Genet A 2016;170:1510-9.

7. Krishna P, Chakraborty RK. Jervell and Lange Nielsen

Cite this article as: Zhang R, Ding C, Wang H. Treatment on arrhythmia electric storm in a Jervell and Lange-Nielsen syndrome patient by ablation of the triggering premature ventricular contraction: a case report. Ann Palliat Med 2021;10(4):4938-4943. doi: 10.21037/apm-19-460a
Syndrome. StatPearls, StatPearls Publishing, 2020;28.

8. Schwartz PJ, Spazzolini C, Crotti L, et al. The Jervell and Lange-Nielsen syndrome: natural history, molecular basis, and clinical outcome. Circulation 2006;113:783-90.

9. Sato A, Chinushi M, Sonoda K, et al. Benign premature ventricular complexes from the right ventricular outflow tract triggered polymorphic ventricular tachycardia in a latent type 2 LQTS patient. Intern Med 2012;51:3261-5.

10. Vojdani S, Amirsalari S, Milanizadeh S, et al. Mutation Screening of KCNQ1 and KCNE1 Genes in Iranian Patients With Jervell and Lange-Nielsen Syndrome. Fetal Pediatr Pathol 2019;38:273-81.

11. Barhanin J, Lesage F, Guillemare E, et al. K(V)LQT1 and lsK (minK) proteins associate to form the $\mathrm{I}(\mathrm{Ks})$ cardiac potassium current. Nature 1996;384:78-80.

12. Lo CF, Numann R. Independent and exclusive modulation of cardiac delayed rectifying $\mathrm{K}+$ current by protein kinase C and protein kinase A. Circ Res 1998;83:995-1002.

13. Schroeder BC, Waldegger S, Fehr S, et al. A constitutively open potassium channel formed by KCNQ1 and KCNE3. Nature 2000;403:196-9.

14. Splawski I, Tristani-Firouzi M, Lehmann MH, et al. Mutations in the hminK gene cause long QT syndrome and suppress IKs function. Nat Genet 1997;17:338-40.

15. Lai LP, Deng CL, Moss AJ, et al. Polymorphism of the gene encoding a human minimal potassium ion channel (minK). Gene 1994;151:339-40.

16. Faridi R, Tona R, Brofferio A, et al. Mutational and phenotypic spectra of KCNE1 deficiency in Jervell and Lange-Nielsen Syndrome and Romano-Ward Syndrome. Human Mutation 2019;40:162-76.

17. Früh A, Siem G, Holmstrom H, et al. The Jervell and Lange-Nielsen syndrome; atrial pacing combined with ss-blocker therapy, a favorable approach in young highrisk patients with long QT syndrome? Heart Rhythm 2016;13:2186-92. 